\title{
Outcomes of multisystem inflammatory syndrome in children temporally related to COVID-19: a longitudinal study
}

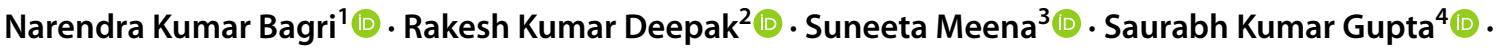

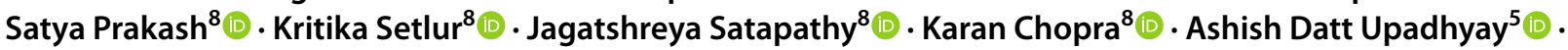

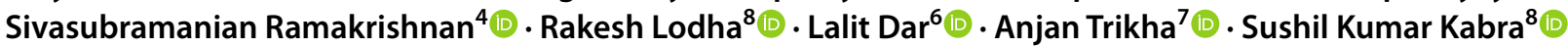

Received: 29 July 2021 / Accepted: 11 October 2021 / Published online: 19 October 2021

(c) The Author(s), under exclusive licence to Springer-Verlag GmbH Germany, part of Springer Nature 2021

\begin{abstract}
To study the clinical, laboratory characteristics and outcomes of multisystem inflammatory syndrome in children (MIS-C) temporally related to coronavirus disease 2019 (COVID-19) in a resource-limited setting. All children meeting the World Health Organization case definition of MIS-C were prospectively enrolled. Baseline clinical and laboratory parameters were compared between survivors and non-survivors. Enrolled subjects were followed up for 4-6 weeks for evaluation of cardiac outcomes using echocardiography. The statistical data were analyzed using the stata- 12 software. Thirty-one children with MIS-C were enrolled in an 11-month period. Twelve children had preexisting chronic systemic comorbidity. Fever was a universal finding; gastrointestinal and respiratory manifestations were noted in $70.9 \%$ and $64.3 \%$, respectively, while $57.1 \%$ had a skin rash. Fifty-eight percent of children presented with shock, and $22.5 \%$ required mechanical ventilation. HSP like rash, gangrene and arthritis were uncommon clinical observations. The median duration of hospital stay was 9 (6.5-18.5) days: four children with preexisting comorbidities succumbed to the illness. The serum ferritin levels (ng/ml) [median (IQR)] were significantly higher in non-survivors as compared to survivors $[1061(581,2750)$ vs $309.5(140,720.08), p$ value $=0.045]$. Six patients had coronary artery involvement; five recovered during follow-up, while one was still admitted. Twenty-six children received immunomodulatory drugs, and five improved without immunomodulation. The choice of immunomodulation (steroids or intravenous immunoglobulin) did not affect the outcome. Most children with MIS-C present with acute hemodynamic and respiratory symptoms. The outcome is favorable in children without preexisting comorbidities.Raised ferritin level may be a poor prognostic marker. The coronary outcomes at follow-up were reassuring.
\end{abstract}

Keywords MIS-C $\cdot$ SARS-CoV-2 $\cdot$ Coronary artery outcomes $\cdot$ MIS-C and Kawasaki disease

Narendra Kumar Bagri

drnarendrabagri@yahoo.co.in

1 Division of Pediatric Rheumatology, Department of Pediatrics, All India Institute of Medical Sciences, New Delhi, India

2 Department of Transplant Immunology and Immunogenetics, All India Institute of Medical Sciences, New Delhi, India

3 Department of Laboratory Medicine, All India Institute of Medical Sciences, New Delhi, India

4 Department of Cardiology, All India Institute of Medical Sciences, New Delhi, India

5 Department of Biostatistics, All India Institute of Medical Sciences, New Delhi, India

6 Department of Microbiology, All India Institute of Medical Sciences, New Delhi, India

7 Department of Anesthesiology and Critical Care, All India Institute of Medical Sciences, New Delhi, India

8 All India Institute of Medical Sciences, New Delhi, India

\section{Introduction}

Multisystem inflammatory syndrome following COVID-19 infection from various pediatric clusters was first reported in January 2020 from Europe and North America [1-3]. Following these reports, the World Health Organization (WHO) circulated a preliminary case definition for multisystem inflammatory disorder temporally associated with COVID in children and adolescents (MIS-C) for reporting surveillance and outlining treatment strategies for this disorder [4]. This entity is also known as Multisystem inflammatory syndrome in children associated with COVID-19and Pediatric Multisystem inflammatory syndrome temporally associated with SARS-CoV-2(PIMS-TS) [5, 6].

The clinical spectrum of MIS-C includesmild persistent febrile illness, Kawasaki diseases like illness and severe MIS-C presenting with shock and multiorgan involvement [7]. The severe form of MIS-C is a life-threatening disease, 
with a mortality of $2 \%$, as reported from a large series of 518 children from the US [8]. Its resemblance to Kawasaki disease also poses a risk of cardiac and coronary involvement to children with MIS-C, with $8-24 \%$ of the cases developing coronary artery abnormalities [9]. However, there is a paucity of data on the outcome of MIS-C fromresource-poor settings. In contrast to the developed world, resource-poor settings arechallenged withfactors like access to health care facilities and the availability of costly immunomodulatory drugs (IVIG and biologicals), which might affect the outcome in such settings.In addition, the high background rate of infections in such settings presentsclinical challenges in differentiating MIS-C from close mimickers like bacterial sepsis and toxic shock syndrome [10]. Considering these facts, we conducted a single center prospective longitudinal study to describe the clinical, laboratory,echocardiographic findings and follow-up of children with MIS-C in a resourcelimited setting.

\section{Methodology}

The present study describes the data from 15-07-2020 to 02-06-2021 of a prospective longitudinal study from a tertiary care teaching hospital: the All India Institute of Medical Sciences, New Delhi, India. Children (less than 15 years of age) with fever $\geq 3$ days were screened for MIS-C. Those meeting the surveillance definition as per WHO [4] were enrolled for this study after receiving written informed consent from parent/guardian. Children with alternative diagnoses were excluded.All children meeting inclusion criteria were enrolled for the study after taking informed consent. The study was approved by the Institute Ethics Committee (IEC-580/19.06.2020, RP-04/2020).

Evidence of current or preceding SARS-CoV-2 infection was demonstrated either through positive naso-and oro-pharyngeal swab for SARS-CoV-2 nucleic acid using reversetranscriptase quantitative PCR (RTPCR)/cartridge-based nucleic acid amplification test (CBNAAT) assay or positive serology for antibody ( $\mathrm{IgG} / \mathrm{IgM}$ ) using ECLIA (electrochemiluminescence) assay on Elecsys anti-SARS-CoV-2on COBAS 6000 (Roche, Switzerland).

Baseline demographic and clinical data werecollected in a predesigned proforma incorporating the details of clinical, laboratory and echocardiography parameters. The enrolled subjects were managed on broad principles provided by the American College of Rheumatology [11]. The inflammatory markers, markers of myocardial inflammation (Troponin/NT-Pro-BNP), cytokine levels (IL- $2,6,18$, TNF- $\alpha$, INF- $\gamma$ ) in addition to the other laboratory parameters were compared with clinical outcome.The studied cytokines were assayed in serum with Bead-based Multiplex Fluorescent Assay (Luminex ${ }^{\mathrm{TM}}$ technology).
The study subjects were followed up for 4-6 weeks. A 2D- echocardiography was performed at 2 and 4-6 weeks after enrollment.Coronary artery abnormalities were represented as $z$ scores [12]. We also estimated the prevalence of macrophage activation syndrome (MAS) as per the 2016 MAS classification criteria [13].

The data were analyzed using the Stata-12 software. Descriptive statistics was obtained for all study variables. Continuous data were expressed as mean (SD) or median and inter-quartile range [IQR] values. The normality in distribution of data was assessed using the shapriowilk test. Categorical data were expressed as proportions. $\chi^{2}$ test was used to compare categorical variables. Student $t$-test was used for comparing normally distributed data, and Mann-Whitney $U$ test was used for comparing non-parametric data. Receiving operative curve analysis was used to find the discriminant ability of serum ferritin and appropriate cutoff for predicting in-hospital mortality. A $p$ value less than 0.05 was considred statistically significant.

\section{Results}

We enrolled 31 children (19 boys) meeting the WHO criteria of MIS-C [4] during thestudy period. The demographic and laboratory characteristics of the study population has been depicted in Table 1 . The mean duration of symptoms was 6.25 days. Twelve children $(38.7 \%)$ had preexisting chronic comorbidities [systemic lupus erythematosus $(n=1)$, Down Syndrome with Tetralogy of Fallot $(n=1)$, Transposition of Great Arteries $(n=1)$, rheumatic heart disease $(n=1)$, acute lymphoblastic leukemia $(n=1)$, chronic renal disorder $(n=2)$, and neurological (seizure disorder $(n=4)$, tubercular meningitis $(n=1)]$. Fever was a universal finding, whereas gastrointestinal (pain abdomen, vomiting and diarrhea) and respiratory symptoms were observed in $71 \%$ and $64.3 \%$, respectively. Eighteen children $(58.06 \%)$ presented with shock, thirteen of whomrequired vasoactive drug support. Seven children (22.5\%) required mechanical ventilation, whereas 13 subjects required respiratory support either through a face mask, nasal cannula or high flow nasal cannula. The maculopapular erythematous rash was the most commonly observed skin lesion. We also observed palpable purpura akin to Henoch-Schonlein purpura in a 13-year-girl and gangrene involving the penis and anterolateral aspect of the left leg in an 8-year boy (Fig. 1). Arthritis involving bilateral knee joints was observed in one girl. The inflammatory markers (ESR, CRP and procalcitonin), serum ferritin, IL-6 and cardiac biomarkers (NT-pro BNP) were raised in the study population (Table1). 
Table 1 Demographic and laboratory characteristics of children with MIS-C

\begin{tabular}{|c|c|}
\hline Parameter & $n=31$ \\
\hline Gender, male $(\%)$ & $19(61.29)$ \\
\hline Age in months, median(IQR), range & $96(60,131), 3-181$ \\
\hline Duration of illness in days, mean (SD) & $6.9(5.7)$ \\
\hline Duration of hospital stay in days, median(IQR) & $9(6.5,18.5)$ \\
\hline Rash, $n(\%)$ & $16(57.14)$ \\
\hline Gastrointestinal symptoms, $n(\%)$ & $22(70.96)$ \\
\hline Respiratory symptoms, $n(\%)$ & $21(67.74)$ \\
\hline Hypotension, $n(\%)$ & $18(58.06)$ \\
\hline Non-purulent conjunctivitis, $n(\%)$ & $8(25.80)$ \\
\hline Mechanical ventilation, $n(\%)$ & $7(22.5)$ \\
\hline \multicolumn{2}{|l|}{ Laboratory characteristics } \\
\hline Either RTPCR/ CBNAAT, $n(\%)$ & $10(32.26)$ \\
\hline Only antibody, $n(\%)$ & $16(51.61)$ \\
\hline Both RTPCR/ CBNAAT and antibody positive & $5(16.13)$ \\
\hline $\mathrm{Hb}(\mathrm{g} / \mathrm{dL})$, median $(\mathrm{IQR})$ & $9.31(8.1,10.9)$ \\
\hline Total leucocyte count $\left(/ \mathrm{mm}^{3}\right)$, median (IQR) & $12,920(8760,22,400)$ \\
\hline Neutrophils \%, median (IQR) & $68(46.3,84.8)$ \\
\hline Lymphocytes \%, median (IQR) & $23(9.3,35)$ \\
\hline Platelet $\operatorname{count}\left(\times 10^{5} / \mathrm{mm}^{3}\right)$, median (IQR) & $3.48(1.8,2.6)$ \\
\hline ESR (mm/h), median (IQR) & $47(36,90)$ \\
\hline $\mathrm{CRP}(\mathrm{mg} / \mathrm{dl})$, median (IQR) & $12.8(3.0,25.9)$ \\
\hline Serum procalcitonin (ng/ml), median (IQR) & $8.39(1.49,31.89)$ \\
\hline Serum ferritin(ng/ml), median (IQR) & $411.05(195.47-1031.93)$ \\
\hline D-dimer (ng/ml), median (IQR) & $3100(500,5250)$ \\
\hline Serum fibrinogen (mg/dL), median (IQR) & $411.45(311.16,466.63)$ \\
\hline Serum triglycerides (mg/dL), median (IQR) & $218(125,406)$ \\
\hline SGOT (U/L), median (IQR) & $41(26,59)$ \\
\hline SGPT (U/L), median (IQR) & $28(15,51)$ \\
\hline Blood urea (mg/dL), median (IQR) & $38(19,68)$ \\
\hline Serum creatinine $(\mathrm{mg} / \mathrm{dL})$, median $(\mathrm{IQR})$ & $0.5(0.3,1.06)$ \\
\hline NT-pro BNP (pg/mL), median (IQR) (normal < 125 pg/mL) & $\begin{array}{l}296.52(24.66,4631.31) \\
(n=15)\end{array}$ \\
\hline \multicolumn{2}{|l|}{ Cytokine profile } \\
\hline IL-6 (pg/mL), median (IQR) $(0.02-10$ pg/mL) & $\begin{array}{l}59.52(12.9,194) \\
(n=23)\end{array}$ \\
\hline IL-18 (pg/mL), median (IQR) (9-812 pg/mL) & $\begin{array}{l}7.59(0.87,94.89) \\
(n=12)\end{array}$ \\
\hline IL-2 (pg/mL), median (IQR) (0.03-90 pg/mL) & $\begin{array}{l}8.92(4.21,53.38) \\
(n=12)\end{array}$ \\
\hline INF- $\gamma(\mathrm{pg} / \mathrm{mL})$, median (IQR) $(0.6-124$ pg/mL) & $\begin{array}{l}7(7,29.93) \\
(n=12)\end{array}$ \\
\hline TNF- $\alpha(\mathrm{pg} / \mathrm{mL})$, median (IQR) $(0.10-98$ pg/mL) & $\begin{array}{l}2.5(2.5,36.25) \\
(n=12)\end{array}$ \\
\hline
\end{tabular}

Four children $(12.9 \%)$ with multiorgan failure in our cohort succumbed to illness. All of these were RT- PCR positive and had preexisting chronic illnesses [chronic kidney disease $(n=1)$, seizure disorder $(n=1)$, Downs syndrome with Tetralogy of Fallot $(n=1)$, rheumatic heart disease $(n=1)]$. Twenty-five children were discharged, and twowere still admitted at the time of drafting this manuscript.
Table 2 compares the various clinical and laboratory parameters in survivors versus those deceased.The serum ferritin levels[median (IQR)] were significantly higher in non-survivors as compared to survivors $[1061(581,2750)$ vs 309.5 $(140,720.08) \mathrm{ng} / \mathrm{mL}, p$ value $=0.045]$. The area under the receiver operator characteristic curve for serum ferritin for in-hospital mortality was 0.82 (95\%CI 0.64-0.99) (Online 
Fig. 1 a Bilateral non-purulent conjunctivitis seen in a 6-yearold boy. b Generalized erythematous rash noted over the abdomen in a 7-year-old boy. c Henoch Schonlein purpura-like non-blanchable palpable purpura seen over bilateral lower limbs in a 13-year-old girl, d, e Gangrene involving penis and anterolateral aspect of left leg in an 8-year-old boy
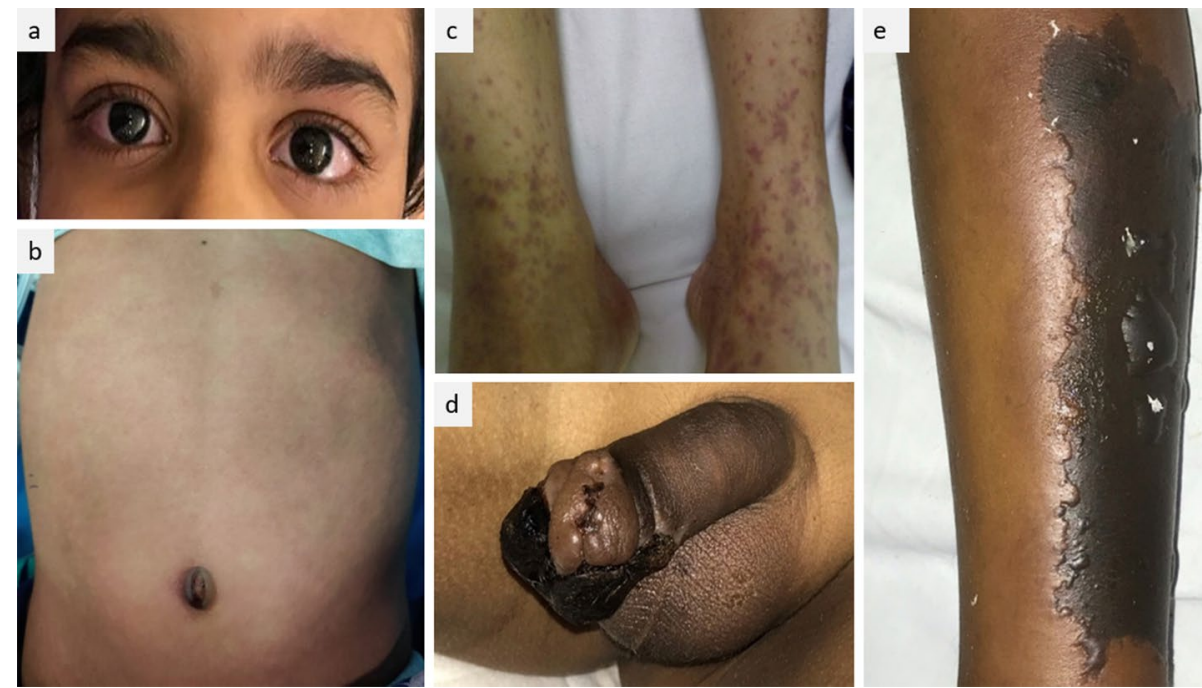

Table 2 Comparison of clinical,laboratory parameters and treatment modalities based on mortality in children with MIS-C

\begin{tabular}{|c|c|c|c|}
\hline & Survivors $(n=27)$ & Non-survivors $(n=4)$ & $p$ value \\
\hline Age, Age in months, median(IQR) & $96(60,120)$ & $130.5(72,161)$ & 0.26 \\
\hline Shock, $n(\%)$ & $14(51.85)$ & $4(100)$ & 0.12 \\
\hline Gastrointestinal symptoms, $n(\%)$ & $18(66.66)$ & $4(100)$ & 0.29 \\
\hline Respiratory symptoms, $n(\%)$ & $17(62.96)$ & $4(100)$ & 0.29 \\
\hline Skin rash, $n(\%)$ & $17(62.96)$ & 0 & 0.032 \\
\hline Pre-existing comorbidity, $n(\%)$ & $8 / 27$ & $4 / 4$ & 0.189 \\
\hline \multicolumn{4}{|l|}{ Laboratory parameters } \\
\hline Total leucocyte count, median (IQR) & $13,240(7700,20,000)$ & $19,685(12,450,27,285)$ & 0.51 \\
\hline Platelet count $\left(\times 10^{5} / \mathrm{mm}^{3}\right)$, median $(\mathrm{IQR})$ & $2.05(1.5,3.46)$ & $0.46(0.17,2.56)$ & 0.098 \\
\hline Serum IL-6 (5.00-15.0 pg/mL), median (IQR) & $41.84(12.9,194)(n=19)$ & $55.06(27.06,328.76)(n=4)$ & 0.21 \\
\hline Serum ferritin $(12-300 \mathrm{ng} / \mathrm{ml})$, median (IQR) & $309.5(140,720.08)$ & $1061(581,2750)$ & 0.045 \\
\hline NT-ProBNP $(\mathrm{pg} / \mathrm{mL})$, median (IQR) $(<125 \mathrm{pg} / \mathrm{ml})$ & $248.63(24.66,3189.7)(n=13)$ & $6078.29(1500.04,10656.5)(n=2)$ & 1.46 \\
\hline D-dimer (ng/ml), median (IQR) $(0.00-255.00 \mathrm{ng} / \mathrm{ml})$ & $1050(741.05,5056)(n=17)$ & $4285(385,14,035)(n=4)$ & 0.16 \\
\hline \multicolumn{4}{|l|}{ Immunomodulation therapy } \\
\hline Supportive care only $(n=5)$ & 5 & 0 & 0.137 \\
\hline Only IVIG $(n=6)$ & 5 & 1 & \\
\hline Only steroids $(n=5)$ & 4 & 1 & \\
\hline Steroid plus tocilizumab $(n=1)$ & 0 & 1 & \\
\hline IVIG plus steroids $(n=14)$ & 13 & 1 & \\
\hline
\end{tabular}

Bold represents a statistically significant value $(p \leq 0.05)$

resource 1). At a cutoff value of $>622 \mathrm{ng} / \mathrm{ml}$, the sensitivity and specificity were $75 \%$ and $74.1 \%$, respectively.Among the 28 children for whom all laboratory parameters for MAS were available, $5(17.9 \%)$ met the classification for MAS; 2 out of 5 children with MAS succumbed to illness.

Table 3 summarizes the available echocardiography findings of the study population. Coronary artery abnormalities were detected in six children (19.4\%); all, except one, resolved at the last follow-up.In our cohort, 8 (25.8\%) children met the classification criteria for Kawasaki disease [classical $(n=1)$, atypical $(n=7)]$.
The various immunomodulatory drugs used in the study population are depicted in Table 2 . Two children were referred to us after receiving immunomodulation [only steroids $(n=1)$, intravenous immunoglobulins (IVIG), plus steroids $(n=1)$ ]. IVIG was administered at a dose of $2 \mathrm{~g} /$ $\mathrm{kg}$. The infusion was withheld midway after $1 \mathrm{~g} / \mathrm{kg}$ due to a transfusion reaction in one child. High dose pulse methylprednisolone (10-30 mg/kg/day for 3 days) followed by oral steroids was administered to 4 subjects.Oral steroids (prednisolone @ dose of $2 \mathrm{mg} / \mathrm{kg} /$ day) were gradually tapered over 2-3 weeks. Two children required a prolonged course 
Table 3 Baseline and follow-up echocardiography in children with MIS-C

\begin{tabular}{|c|c|c|c|}
\hline Echo parameter & Baseline & Echo at 2-week follow-up & $\begin{array}{l}\text { Echo at } 4 \text { to } \\
\text { 6-week follow-up }\end{array}$ \\
\hline $\begin{array}{l}\text { Number of children with low ejection } \\
\text { fraction }(\mathrm{EF} \leq 55 \%), n\end{array}$ & $13 / 29$ & $1 / 16$ & $4 / 12$ \\
\hline Ejection fraction, mean (SD) & $55.75(9.24)$ & $59.18(7.97)$ & $57.75(4.45)$ \\
\hline Coronary artery abnormalities, $n$ & $\begin{array}{l}\mathrm{RCA}^{*}: 2 / 19 \\
\mathrm{LMCA}^{\prime} 0 / 20 \\
\mathrm{LAD}^{\#}: 1 / 11\end{array}$ & $\begin{array}{l}\text { RCA: } 0 / 14 \\
\operatorname{LMCA}^{\$}: 1 / 12 \\
\operatorname{LAD}^{\$}: 1 / 10 \operatorname{LCx}^{\$}: 1 / 12\end{array}$ & $\begin{array}{l}\text { RCA: } 0 / 12 \\
\text { LMCA:0/12 } \\
\text { LAD:0/4 }\end{array}$ \\
\hline Any other & $\begin{array}{l}\text { LCx wall thickening }(n=1) \\
\text { Peri-vascular brightness }(n=1)\end{array}$ & Peri-vascular brightness $(n=1)$ & - \\
\hline Valvular regurgitation & $\begin{array}{l}\text { Mild MR and TR: } 3 / 28 \\
\text { Mild MR: } 1 / 28 \\
\text { Mild TR: } 1\end{array}$ & Mild MR and TR $(n=1)$ & Mild TR $(n=2)$ \\
\hline Pericardial effusion, $\mathrm{n}$ & Mild $(n=1)$ & 0 & 0 \\
\hline Any other abnormality & - & - & Mild PAH $(n=1)$ \\
\hline
\end{tabular}

Z-score

One child had RCA $z$ score of $* 2.15$

One child had z scores of RCA and LAD 3.17 and ${ }^{\#} 2.53$ respectively

${ }^{\$}$ Same child, LAD: 5.2, LMCA and LCx dilated

Dilation or Aneurysm based on Z scores

Dilation ( $z$ score 2-2.5)

Small ( $z$ score $2.5-5)$

Medium ( $z$ score 5-10)

Giant $(z$ score $>10)$

$L A D$ Left Anterior Descending artery, $L M C A$ Left Main Coronary artery, $L C x$ Left Circumflex artery, TR Tricuspid Regurgitation, $M R$ Mitral Regurgitation, $P A H$ Pulmonary Arterial Hypertension

(> 3 months) of oral prednisolone due to the possibility of evolving rheumatic disorder. There was no statistically significant difference in mortality based on the choice of immunomodulatory agent ( $p$ value $=0.137$ ). Twelve subjects received aspirin in antiplatelet doses for 4-6 weeks. In addition, two children required enoxaparin: one with low ejection fraction received it for a week while the other with penile gangrene and venous thrombosis (involving right saphenofemoral junction and deep veins of the calf in bilateral lower limbs) was still on enoxaparin at the last follow-up at 12 week. All children also received empirical broad-spectrum antibiotics; however, the blood cultures were sterile in all subjects.

\section{Discussion}

This study reports the clinical, laboratory profile and outcomes at short-term follow-up of children with MIS-C from India. During the study period, 31 children with MIS-C were enrolled. In addition to the usual clinical features, HSP like rash, penile gangrene and arthritis were few uncommon findings in our cohort. Baseline coronary artery abnormalities were observed in $6(19.4 \%)$ subjects, which resolved in all except one at the last follow-up. Four children (12.9\%) with underlying chronic comorbidities succumbed to the illness.

All our subjects were either RTPCR/ CBNAAT positive or antibody positive, unlike other series from tropical countries wherein children with a history of contact (without laboratory evidence to support an association with SARSCoV-2) were also enrolled (27\% in Dhanalakshmi et al. and $34 \%$ in Jain et al. series) [14, 15]. In the authors' opinion, laboratory evidence of SARS-CoV-2 infection adds strength to the inclusion criteria while classifying febrile children as MIS-C and may avoid over-diagnosis of MIS-C. This is more relevant to tropical countries where the background infections mimicking MIS-C are widely prevalent.

Similar to the existing literature, the spectrum of illness in our series varied from non-life-threatening febrile episodes to severe illness resulting in cardiovascular collapse and shock [16]. Nearly $60 \%$ of our study population presented with shock, comparable to various series from different parts of the globe $[3,17]$. In $64 \%$ of children, respiratory symptoms were seen, two-thirds of whom required non-invasive oxygen support, while a third of these required mechanical ventilation. Gastrointestinal symptoms (mild to moderate severity) were seen in around $71 \%$ of children. Twelve of the 31 children had underlying 
systemic comorbidity. Ahmad et al. in their systematic review reported a significant proportion of comorbidities with obesity accounting for half of the associated comorbidities [18], which is in contrast to our series where only $2 / 31(6.45 \%)$ were obese (results not shown).

We also observed few peculiar findings in our series. A 13-year-old girl presented with a rash similar to IgA vasculitis. She also had pain abdomen but did not have other features consistent with IgA vasculitis; neither did she develop recurrence of symptoms or renal involvement during 6 months of follow-up. Allez et al. have previously reported the association of IgA vasculitis with COVID-19 in a young adult with Crohn's disease on anti-TNF [19]. Although we couldn't contemplate skin biopsy in our case, the palpable purpura was clinically similar to HSP. Another girl with an underlying seizure disorder presented with arthritis of bilateral knee joints. The workup for the evolution of other rheumatic diseases, such as lupus, was non-contributory till the last follow-up. These observations are in sync with the emerging evidence of other vasculitis and rheumatic disorders in association with COVID-19 [18], and thus, it is imperative to note such clinical findings and follow these children for the evolution of immune manifestations. An 8-year-old boy developed penile gangrene with deep vein thrombosis requiring prolonged anticoagulation. Thrombosis is frequently reported in adults with COVID-19 but is an uncommon finding in children [20]. Feldstein et al. have reported one child with imaging confirmed symptomatic venous thromboembolism [21]. The index child in our series was aptly managed with immunosuppression and anticoagulants. These gangrenous changes may result from ischemic changes, resulting from vascular insult and necrosis of SARSCoV-2 infected endothelial cells. Kabeerdosset al. have proposed this mechanism for COVID associated chillbains or COVID toes [10]. Timely recognition and management of such organ/limb-threatening thrombotic complications are warranted for a successful outcome.

The median values of laboratory markers of cardiac involvement (NT-pro-BNP) were elevated in the study population (Table 1). Markers of cardiac injury like Troponin and BNP are elevated in 64-95\% of children with MIS-C in various series [17]. Aliased et al. in their review, reported wide variability in left ventricular dysfunction, ranging from $20 \%$ to nearly all, in various series [17]. Coronary artery abnormalities have been reported in $8-24 \%$ of cases in other series [9]. In our series, six patients $(19.3 \%)$ had coronary involvement. There is a scarcity of data about follow-up outcomes. Echocardiography demonstrated a low ejection fraction $(<55 \%)$ in $44.8 \%$ of the subjects, which decreased to $33.3 \%$ at $4-6$ weeks follow-up.The short-term outcome for coronaries was reassuring in our series; the coronary artery abnormalities resolved in 5 out of 6 children.
Among the clinical and laboratory factors studied in our series, serum ferritin levels were significantly higher in those who succumbed. Fifty percent of the children who succumbed to illness had MAS. This finding is consistent with a large retrospective study from the US by Abrams et al. [22], comprising 1080 children wherein ferritin was found to be an indicator of shock and cardiovascular compromise. Serum ferritin may also help in differentiating MIS-C from its close mimicker Kawaski disease; Yener et al. reported higher serum ferritin levels in children with MIS-C as compared to $\mathrm{KD}$ [ $440 \mathrm{ng} / \mathrm{ml}$ versus $170 \mathrm{ng} / \mathrm{ml}$ in MISC and KD respectively] in a multicentric Turkish study, enrolling 154 children with MIS-C and 59 children with KD [23]. Similar to Yener et al. study, we also obsereved an elevated serum ferritin levels [median $411.45(311.16,466.63) \mathrm{ng} / \mathrm{ml})$ ] in our cohort.

We also observed raised levels of IL-6 in the mortality group as compared to survivors; however, the difference was not statistically significant, unlike Abrams et al. series describing an association of increased risk of hemodynamic compromisewith IL-6 levels; the small sample size in the present series might have resulted in varying observations.

The high mortality rate $(12.9 \%)$ observed in the present series is in stark contrast to that reported from multi-hospital data from US [8, 21]. The reported mortality in Indian series ranges from 0 to $27.5 \%$ [14, 15, 24]. All children who succumbed to illness in our series had underlying comorbidities, which might have contributed to poor outcomes. The only series reporting higher mortality $(27.5 \%)$ than ours, by Maheshwari et al. didn't mention about the underlying comorbidities. The other speculated factors contributing toincreased mortality in resource-poor settings include delayed referral to tertiary care centers and delay in instituting timely immunomodulation [24].

Our study has certain limitations, including a small sample size, which precludes us from undertaking a subgroup analysis in children receiving various immunomodulatory drugs. In addition, our center, a tertiary care referral hospital, may not reflect the overall spectrum of MIS-C, and the results may not be generalizable. Although the mortality is high compared to other series, the absolute number is small to make meaningful conclusions on the predictors of mortality.

\section{Conclusion}

The underlying hyper-inflammation in MIS-C causes acute hemodynamic deterioration in most children.Children with MIS-C can present with unusual findings such as HSP like rash, gangrene and arthritis. In children without any premorbid conditions, the outcome is usually optimistic.Raised ferritin level may be a poor prognostic marker. The follow-up at 
4-6 weeks for coronary artery outcomes is reassuring.Large multi-centric follow-up studies are required to delineate the best therapeutic modalities and predictors of outcome; particulary in resource-limited settings.

Supplementary Information The online version contains supplementary material available at https://doi.org/10.1007/s00296-021-05030-y.

Acknowledgements The Research section AIIMS, New Delhi for funding and supporting the study. Bhavi Malik, junior nurse, for her incessant efforts in ensuring the smooth conduct of the study. Dr Lata Singh, Scientist, for her help in carrying out laboratory work.

Author contributions NKB: conceptualization, data acquisition, analysis and drafting the initial manuscript. RL: contributed to study conception and design and supervised the study, and critically appraised the manuscript. SKK: contributed to study conception and provided critical inputs for the draft. SP, KS, JSS and KC: data collection. ADU: provided expertise in statistical analysis. SKG, SR, RKD, LD, AT: reviewed and provided valuable inputs to the draft. All authors reviewed the draft and approved the final version of the manuscript.

Funding Intramural project, All India Institute of Medical Sciences, New Delhi.

\section{Declarations}

Conflict of interest All authors declare that they have no conflict of interest.

Ethical approval Approved by the institute ethics committee (IEC580/19.06.2020, RP-04/2020)

Data availability All data and material are available with the corresponding author.

Code availability Available with the corresponding author.

Consent for participation Consent obtained from the parents of all enrolled children at the time of enrollment in the prescribed participation consent form.

Consent for publication Consent obtained from the parents of all enrolled children, as well as the authors.

\section{References}

1. Liu W, Zhang Q, Chen J, Xiang R, Song H, Shu S et al (2020) Detection of Covid-19 in children in early January 2020 in Wuhan, China. N Engl J Med 382(14):1370-1371

2. Belhadjer Z, Méot M, Bajolle F, Khraiche D, Legendre A, Abakka $S$ et al (2020) Acute heart failure in multisystem inflammatory syndrome in children (MIS-C) in the context of global SARSCoV-2 pandemic. Circulation 142(5):429-436

3. Verdoni L, Mazza A, Gervasoni A, Martelli L, Ruggeri M, Ciuffreda $M$ et al (2020) An outbreak of severe Kawasaki-like disease at the Italian epicentre of the SARS-CoV-2 epidemic: an observational cohort study. Lancet 395:1771-1778

4. World Health Organization. Multisystem inflammatory syndrome in children and adolescents with COVID-19. Scientific brief (2020). (https://www.who.int/publications/i/item/ multisystem-inflammatory-syndrome-in-children-and-adole scents-with-covid-19)

5. Centers for Disease Control and Prevention. Emergency preparedness and response: multisystem inflammatory syndrome in children (MIS-C) associated with coronavirus disease 2019 (COVID-19). Health advisory (2019). (https://emergency.cdc. gov/han/2020/han00432.asp. opens in new tab)

6. Royal College of Paediatrics and Child Health. Guidance: paediatric multisystem inflammatory syndrome temporally associated with COVID-19 (2020). https://www.rcpch.ac.uk/resou rces/guidance-paediatric-multisystem-inflammatory-syndrometemporally-associated-covid-19. Accessed 22 May 2020

7. Whittaker E, Bamford A, Kenny J, Kaforou M, Jones CE, Shah $P$ et al (2020) Clinical characteristics of 58 children with a pediatric inflammatory multisystem syndrome temporally associated with SARS-CoV-2. JAMA 324(3):259

8. Son MBF, Murray N, Friedman K, Young CC, Newhams MM, Feldstein LR et al (2021) Multisystem inflammatory syndrome in children-initial therapy and outcomes. N Engl J Med. https://doi.org/10.1056/NEJMoa2102605

9. Valverde I, Singh Y, Sanchez-de-Toledo J, Theocharis P, Chikermane A, Di Filippo S et al (2021) Acute cardiovascular manifestations in 286 children with multisystem inflammatory syndrome associated with COVID-19 infection in Europe. Circulation 143(1):21-32

10. Kabeerdoss J, Pilania RK, Karkhele R, Kumar TS, Danda D, Singh S (2021) Severe COVID-19, multisystem inflammatory syndrome in children, and Kawasaki disease: immunological mechanisms, clinical manifestations and management. Rheumatol Int 41(1):19-32

11. Henderson LA, Canna SW, Friedman KG, Gorelik M, Lapidus SK, Bassiri H et al (2020) American College of rheumatology clinical guidance for multisystem inflammatory syndrome in children associated With SARS-CoV-2 and hyperinflammation in pediatric COVID-19: version 1. Arthritis Rheumatol 72(11):1791-1805

12. McCrindle BW, Li JS, Minich LL, Colan SD, Atz AM, Takahashi $\mathrm{M}$ et al (2007) Coronary artery involvement in children with Kawasaki disease. Circulation 116(2):174-179

13. Ravelli A, Minoia F, Davì S, Horne A, Bovis F, Pistorio A et al (2016) 2016 Classification criteria for macrophage activation syndrome complicating systemic juvenile idiopathic arthritis: a European League Against Rheumatism/American College of Rheumatology/Paediatric Rheumatology International Trials Organisation Collaborative Initiative. Arthritis Rheumatol 68(3):566-576

14. Dhanalakshmi K, Venkataraman A, Balasubramanian S, Madhusudan M, Amperayani S, Putilibai S et al (2020) Epidemiological and clinical profile of pediatric inflammatory multisystem syndrome-temporally associated with SARS-CoV-2 (PIMS-TS) in Indian children. Indian Pediatr 57(11):1010-1014

15. Jain S, Sen S, Lakshmivenkateshiah S, Bobhate P, Venkatesh S, Udani $S$ et al (2020) Multisystem inflammatory syndrome in children with COVID-19 in Mumbai, India. Indian Pediatr 57(11):1015-1019

16. Jiang L, Tang K, Levin M, Irfan O, Morris SK, Wilson $\mathrm{K}$ et al (2020) COVID-19 and multisystem inflammatory syndrome in children and adolescents. Lancet Infect Dis 20(11):e276-e288

17. Alsaied T, Tremoulet AH, Burns JC, Saidi A, Dionne A, Lang SM et al (2021) Review of cardiac involvement in multisystem inflammatory syndrome in children. Circulation 143(1):78-88

18. Ahmed M, Advani S, Moreira A, Zoretic S, Martinez J, Chorath $\mathrm{K}$ et al (2020) Multisystem inflammatory syndrome in children: a systematic review. EClinicalMedicine 26:100527

19. Allez M, Denis B, Bouaziz J, Battistella M, Zagdanski A, Bayart $\mathrm{J}$ et al (2020) COVID-19-Related IgA vasculitis. Arthritis Rheumatol 72(11):1952-1953 
20. Levi M, Thachil J, Iba T, Levy JH (2020) Coagulation abnormalities and thrombosis in patients with COVID-19. Lancet Haematol 7(6):e438-e440

21. Feldstein LR, Tenforde MW, Friedman KG, Newhams M, Rose EB, Dapul H et al (2021) Characteristics and outcomes of US children and adolescents with multisystem inflammatory syndrome in children (MIS-C) compared with severe acute COVID-19. JAMA 325(11): 1074

22. Abrams JY, Oster ME, Godfred-Cato SE, Bryant B, Datta SD, Campbell AP et al (2021) Factors linked to severe outcomes in multisystem inflammatory syndrome in children (MIS-C) in the USA: a retrospective surveillance study. Lancet Child Adolesc Health 5(5):323-331

23. OtarYener G, PaçKısaarslan A, Ulu K, Atalay E, Haşlak F, Ozdel S et al (2021) Differences and similarities of multisystem inflammatory syndrome in children, Kawasaki disease and macrophage activating syndrome due to systemic juvenile idiopathic arthritis: a comparative study. Rheumatol Int 27:1-7. https://doi. org/10.1007/s00296-021-05008-w

24. Maheshwari A, Mahto D, Kumar V, Gulati S, Pemde H, Saha A et al (2021) Comparison of clinical and laboratory profile of survivors and non-survivors of SARS-CoV-2-related multisystem inflammatory syndrome of childhood in India: an observational study. J Paediatr Child Health. https://doi.org/10.1111/jpc.15675

Publisher's Note Springer Nature remains neutral with regard to jurisdictional claims in published maps and institutional affiliations. 